\title{
Smart Walking Stick for the Visually Challenged
}

\author{
Mohammed Ahsan Kollathodi, Rishav Drolia, Ananda Kumar S \\ Department of Computer Science and Engineering, Vellore Institute of Technology
}

\begin{tabular}{l}
\hline Article Info \\
\hline Article history: \\
Received Jun 1, 2018 \\
Revised Jul 14, 2018 \\
Accepted Aug 21, 20 \\
\hline Keywords: \\
Cellular (GSM) \\
Internet of Things \\
Microcontroller \\
Visually Challenged
\end{tabular}

\begin{abstract}
Internet of things mixed with Radio Frequency identification generation permit a whole new context for whole new devices that are capable of combining their bodily and virtual existences. Radio Frequency identification, placing an identification label into every object, allows a clever device to get information, both actual-time or digital-connected statistics, without any physical touch. Information retrieved from such an object, turns it right into a capacity smart object, clearly able to car pick out itself and, if protection problems are certainly handled, maximum in all likelihood capable to connect to the worldwide net. This way, one can get a ubiquitous framework to get admission to, display and manipulate any of these smart items over a web of linked things. RFID tags in scientific context enable a fast and specific identification of each clever entity, permitting a ubiquitous and brief get admission to personal fitness data over an internet of factors [1-2]. People have been struggling with sicknesses weird. Visually challenged human beings are blind people who are very common and difficult to cope with of their way. The principle aim of this project is to aid the visually challenged human beings with a better navigation device. This clever strolling stick is greater state-of-the-art device with many embedded features.
\end{abstract}

Copyright $@ 2018$ Institute of Advanced Engineering and Science. All rights reserved.

\section{Corresponding Author:}

Mohammed Ahsan Kollathodi,

Department of Computer Science and Engineering,

Vellore Institute of Technology, India.

Email: cswebdvlpr@gmail.com

\section{INTRODUCTION}

The trouble of surveillance and target detection has found its vicinity in numerous civilian and army packages, and IoT is properly desirable to cope with this trouble. Radio frequency (RF) sensing strategies are the following generation technology, which offer awesome advantages over conventional manner of sensing used for surveillance and target detection applications of IoT. But, RF sensing techniques haven't begun to be broadly researched due to lack of transmission and computational resources within IoT. Latest advancements in sensing, computing, and conversation technology have made radio detection enabled sensing techniques to be had to IoT. However, massive studies are yet to be done in developing reliable and strength green goal detection algorithms for useful resource restrained IoT. In this paper, we've proposed a multi-sensor RF sensing-primarily based goal detection architecture for IoT [3-4]. The proposed target detection structure is adaptable to interference, that's induced due to the co-life of sensor nodes inside IoT and adopts clever sensing strategies to reliably hit upon the presence of the targets. A waveform selection criterion has been proposed to discover the most appropriate choice of transmit waveforms inside a given set of sensing situations to optimize the goal detection reliability and electricity intake inside the IoT.

Tremendous studies have been committed to the exploration of various technologies together with records technology (IT) in complementing and strengthening present healthcare offerings. Particularly, the Internet of things (IoT) has been broadly carried out to interconnect to be had medical resources and provide reliable, effective and clever healthcare provider to the elderly and sufferers with a continual illness. The 
intention of this paper is to summarize the programs of IoT inside the healthcare industry and discover the feasible trend and directions of destiny studies on this discipline. based on a complete literature review and the dialogue of the achievements of the researchers, the advancement of IoT in healthcare systems had been tested from the perspectives of allowing technologies and methodologies, IoT-based totally clever devices and systems, and various applications of IoT in the healthcare industries. subsequently, the challenges and prospects of the development of IoT based healthcare systems are mentioned in element.

Visually impaired or blindness is the incapacity to see the surroundings. Some are referred to as visually challenged, despite the fact that they can see a little. As a whole, blindness is solely triggered by malnutrition and sickness. Due to illness or accidents the humans have become visually impaired. WHO estimates that eighty percent of visual impairment is either preventable or curable with a complete solution. Two hundred and eighty-five million human beings are expected to be visually impaired in the world.

The advanced stick gives a correct decision of obstacle and is very efficient at guiding a person. Microcontroller is a small low cost single chip pc. It is mainly used to govern alternative electronic devices and acts as an interfacing unit. Ultrasonic sensors are in systems which examine goals by means of deciphering the mediated indicators. The buzzer is ON while an obstacle is detected through an ultrasonic sensor on this device, buzzer is used for varies environment.

Tremendous studies have been committed to the exploration of various technologies together with records technology (IT) in complementing and strengthening present healthcare offerings. Particularly, the Internet of things (IoT) has been broadly carried out to interconnect to be had medical resources and provide reliable, effective and clever healthcare provider to the elderly and sufferers with a continual illness. The intention of this paper is to summarize the programs of IoT inside the healthcare industry and discover the feasible trend and directions of destiny studies on this discipline. based on a complete literature review and the dialogue of the achievements of the researchers, the advancement of IoT in healthcare systems had been tested from the perspectives of allowing technologies and methodologies, IoT-based totally clever devices and systems, and various applications of IoT in the healthcare industries. subsequently, the challenges and prospects of the development of IoT based healthcare systems are mentioned in element [5-12].

In builtintegrated, technology can assist built-in built-ingintegrated many obstacles that is built with disabilities face. Those built-in styles of technology are referred to as assistive generation (AT). There are numerous styles ofdisabilities, built-in bodily disabilities, integrated-impaired, and visually-impaired.

\section{RESEARCH METHOD}

Ultrasonic detector transmits supersonic waves into the air and detects mirrored waves from an object. There can be several applications for supersonic sensors, like in intrusion alarm systems, automatic door openers and backup sensors for cars. Rate of wave propagation is expressed by multiplication of frequency and wavelength and this is attributable to higher resolution, it's potential to induce higher measure creates huge accuracy. Metal, wood, concrete, glass, rubber and paper, etc., mirror close to 100 percent of supersonic waves, these objects are easily detected.

A buzzer is an audio device, which can be a mechanical device, or electronic. Typical users of buzzers and beepers embrace alarms, timers and confirmation of user input like a click or keystroke. Early devices were supported as a mechanical device system similar to a electrical bell.

A DC motor is an electrical machine that converts electrical energy into mechanical power. Most kinds of motors offer rotation. DC motors are often controlled by varied power providers. These are the main components that are required within the project.

Once we have the basic requirements we go creating the required circuit. This is done by wiring the Arduino, we use the mini breadboard to connect the ultrasonic sensor to the Arduino using the Jumper wires.

Some of the parts will have to be directly connected to the Arduino and these include parts such as the buzzer.

Table 1. Different parameters which are involved within the protocol

\begin{tabular}{cccccc}
\hline Technology & $\begin{array}{c}\text { Standard } \\
\text { /Protocol }\end{array}$ & $\begin{array}{c}\text { Transmission } \\
\text { rate }\end{array}$ & $\begin{array}{c}\text { Maximum } \\
\text { distance }\end{array}$ & Frequency bands & $\begin{array}{c}\text { Characteristic / } \\
\text { limitation }\end{array}$ \\
\hline Cellular & GSM , GPRS, & $12-55 \mathrm{Mbps}$ & Max $100 \mathrm{Km}$ & (I) $900-1800 \mathrm{MHz}$ & $\begin{array}{c}\text { Mostly used , here the } \\
\text { wireless spectrum is } \\
\text { limited . }\end{array}$ \\
& HSPA +, LTE & & & (II) $800-2600 \mathrm{MHz}$ & $\begin{array}{c}\text { lited } \\
\end{array}$ \\
& & & & & \\
\hline
\end{tabular}


The Hardware requirements are given as:

a. An Ultra sonic sensor

b. A mini breadboard

c. A 9 Volt battery

d. A 9-volt battery connector

e. DC Male Power Jack

f. A Buzzer

g. Some Jumper Wire

We make connections which include:

Ultrasonic (GND) to Arduino (GND).

Ultrasonic TRIG to Arduino D12.

Ultrasonic ECHO to Arduino D11.

Buzzer RED to Arduino D8.

Buzzer BLACK to Arduino GND.

Ultrasonic VCC to Arduino $5 v$.

9-volt battery RED to Toggle switch pin 1.

9-volt battery BLACK to DC male power jack (-).

Toggle switch pin 2 to DC male power jack (+).

Once we are done with the wiring, we proceed with the coding and for that we upload the code into the Arduino IDE and then sync it with the device. Once we have the code running and working, we go for creating the outer solid body and bringing out the final product.

The Algorithm is given as:

use pin 13 as trigPin

use pin 12 as echoPin

use pin 7 as motor

use pin 6 as buzzer

function setup

\{

set trigPin to OUTPUT

set echoPin to INPUT

set motor to OUTPUT

set buzzer to OUTPUT

\}

function loop

\{

declare duration, distance;

write $L O W$ to trigPin

delay 2 Microseconds

write HIGH to trigPin

delay 10 Microseconds

write LOW to trigPin

duration $=$ read pulse HIGH of echoPin

distance $=($ duration $/ 2) / 29.1$

if (distance $<70)$

\{

write HIGH to motor

write HIGH to buzzer

\}

else

\{

write LOW to motor

write LOW to buzzer

\}

delay 500

\}

The flow diagram for the device is given to be as:

Indonesian J Elec Eng \& Comp Sci, Vol. 12, No. 3, December 2018 : 1282 - 1288 


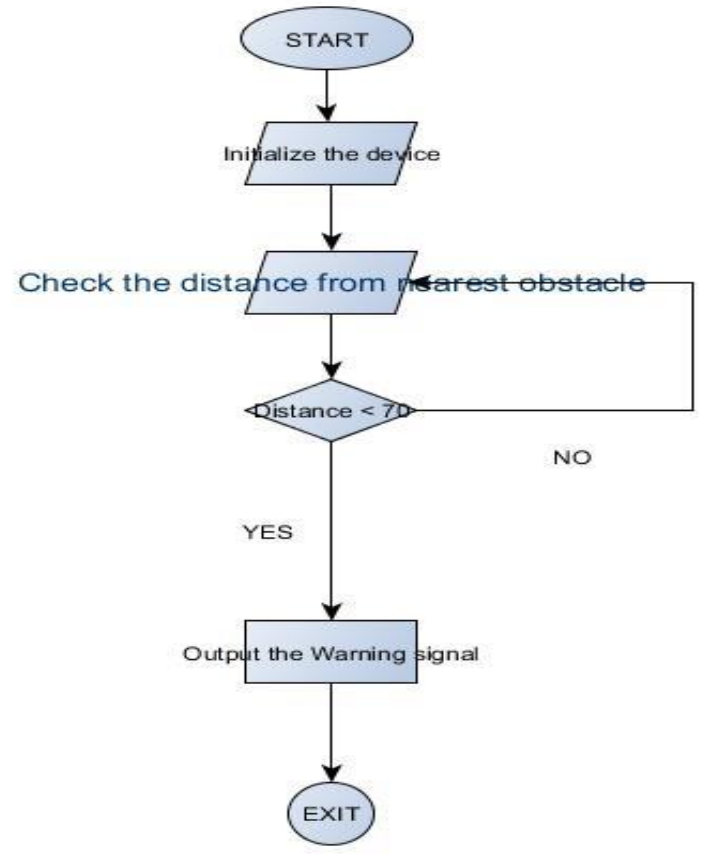

Figure 1. Overall system layout represented as flowchart

The flow diagram for the Warning message notification system is given as:

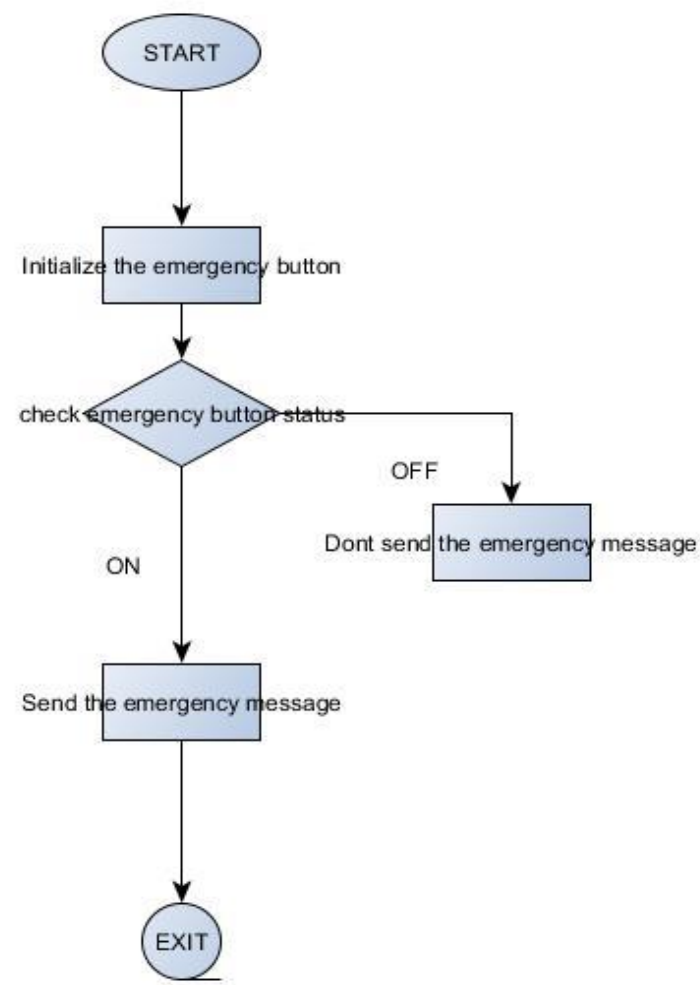

Figure 2. The Emergency feature incorporated within the system 


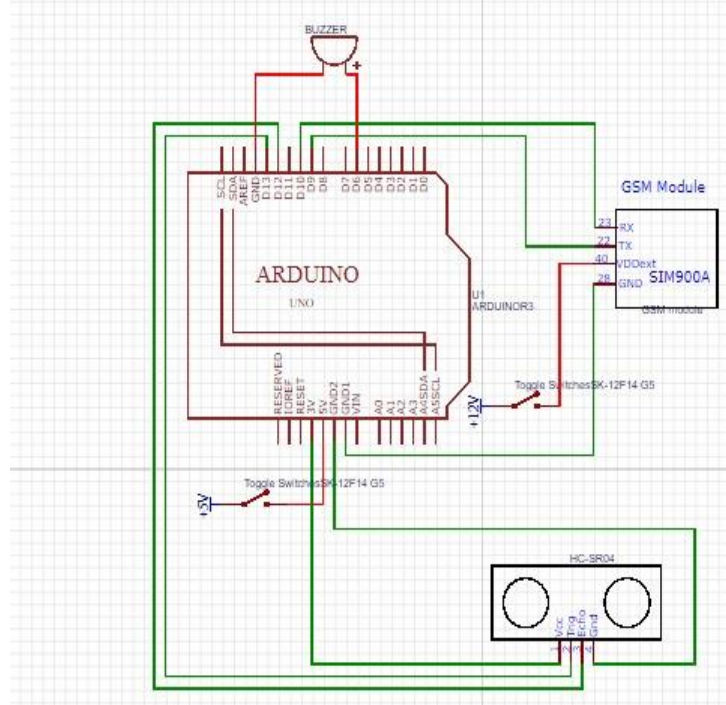

Figure 3. Blueprint of the proposed system

The flow chart for the system as a whole is given as:

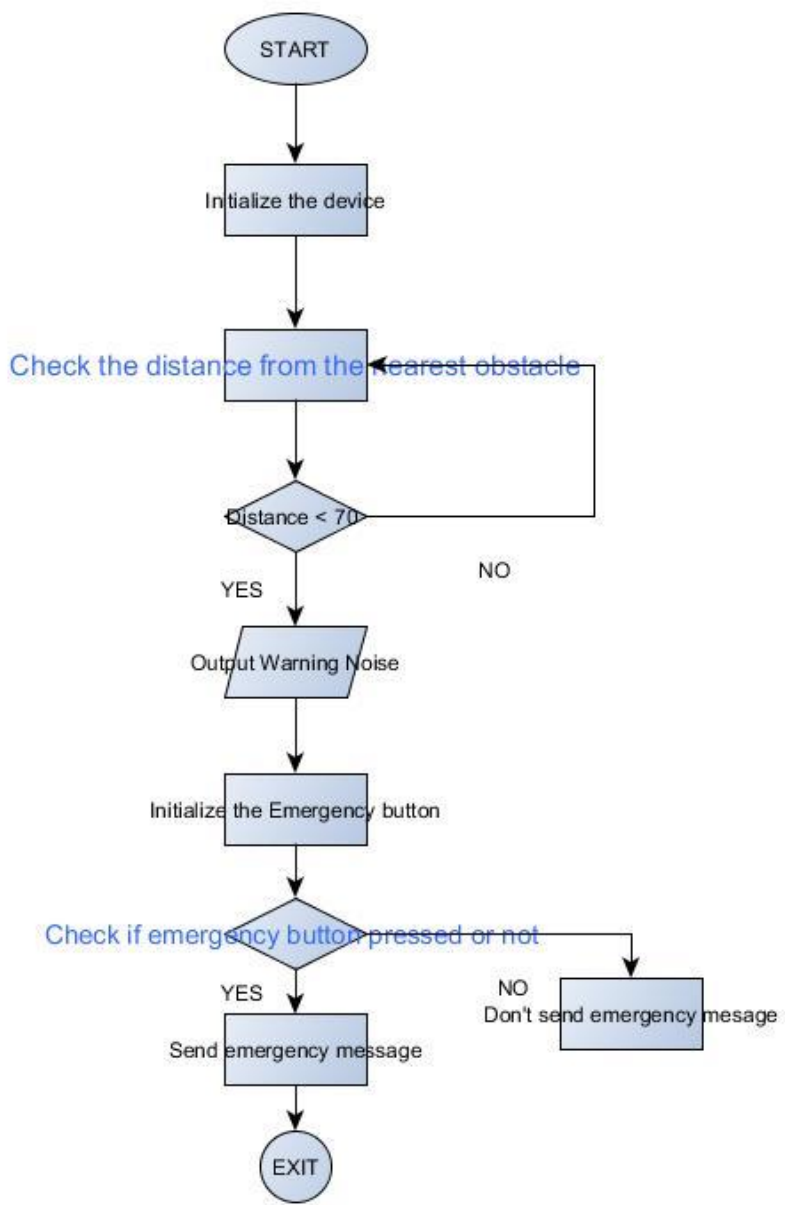

Figure 4. Enhanced system layout represented as flowchart 


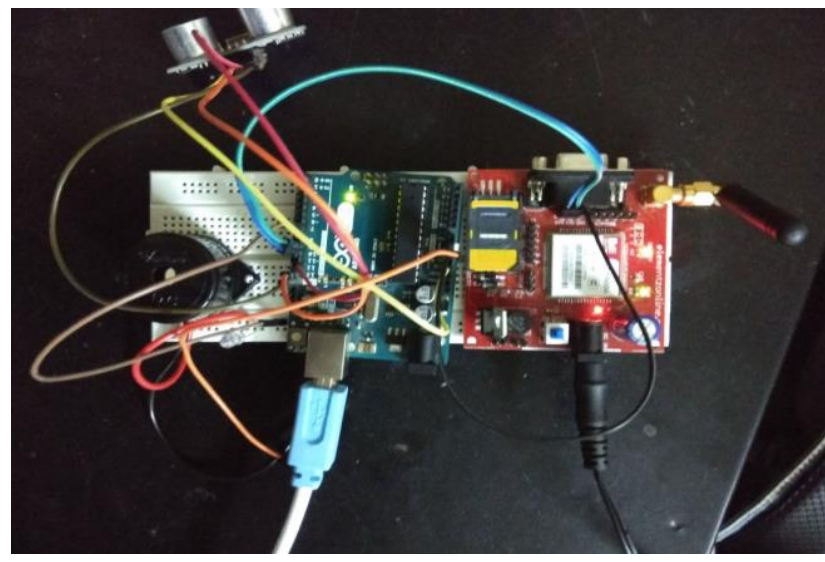

Figure 5. The final implemented circuit

\section{RESULTS AND ANALYSIS}

The circuit was successfully built and created to carry out the required functionalities .From many of the tests that were carried out it was found out that the device did respond well to the user surroundings provided optimal conditions were provided. An emergency situation was simulated and once the button was activated and emergency message was sent to the destination number. The buzzer was found to be very effective in producing signals which was fine tuned to produce good output.

The observation from the trials was found out as Table 2. Signals were also sent to the person in use as audio messages at the same time the buzzer produced a vibration.

Table 2. Final test result after repeated experiments

\begin{tabular}{llll}
\hline Distance $(\mathrm{cm})$ & Status & Emergency button & Message (At destination) \\
\hline$<75$ & Sound was produced & ON & Received \\
$>75$ & Sound was not produced & OFF & Not received \\
\hline
\end{tabular}

\section{CONCLUSION}

The smart walking system can be applied in any kind of terrain, and any form of trail. However there should be a minimum standard size that should be kept for the stick and this should be well handled. The obstacles will be successfully be detected and environmental changes will be identified. With such great advantage people would be able to travel more easily and quickly reaching the destination with ease. With this project we intend to create a very small scale at the same time very much effective device which is cheap and is user friendly interface which is smooth with very less errors ensuring high reliability and efficiency.

In future work many more features can be added such as installing a narrator and also a GPS. However, this system will be very much be adequate and efficient for the user and it is well implemented.

\section{ACKNOWLEDGEMENTS}

This project was partially supported by our institute and it's management, associated members and we would like to thank them for helping us throughout the project .

\section{REFERENCES}

[1] S. K. Bolisetti, M. Patwary, A. H. Soliman, and M. Abdel-Maguid, "RF sensing based target detector for smart sensing within internet of things in harsh sensing environments," IEEE Access, vol. 5, pp. 13346-13363, 2017.

[2] Z. Zou, K. J. Li, R. Li, and S. Wu, "Smart home system based on IPV6 and ZIGBEE technology," Procedia Eng., vol. 15, pp. 1529-1533, 2011.

[3] S. T. V. Jismi Johnson, Nikhal Rajan P, Nivya M Thomas, Rakendh C S, "Smart Walking Stick for Viually," vol. 3, no. 24, pp. 557-560, 2017.

[4] Y. YIN, Y. Zeng, X. Chen, and Y. Fan, "The internet of things in healthcare: An overview," J. Ind. Inf. Integr., vol. 1, pp. 3-13, 2016.

[5] M. Pinto, R. D. Stanley, S. Malagi, V. P. K, and A. K. M. K, "Smart Cane for the Visually Impaired," vol. 7, no. 3, pp. 73-76, 2017. 
[6] A. Santos, J. Macedo, A. Costa, and M. J. Nicolau, "Internet of Things and Smart Objects for M-health Monitoring and Control," Procedia Technol., vol. 16, pp. 1351-1360, 2014.

[7] S. Li, L. Da Xu, and S. Zhao, "The internet of things: a survey," Inf. Syst. Front., vol. 17, no. 2, pp. 243-259, 2015.

[8] S. K. Bolisetti, M. Patwary, A. H. Soliman, and M. Abdel-Maguid, "RF sensing based target detector for smart sensing within internet of things in harsh sensing environments," IEEE Access, vol. 5, pp. 13346-13363, 2017.

[9] F. Razzak, "Spamming the Internet of Things: A possibility and its probable solution," Procedia Comput. Sci., vol. 10, pp. 658-665, 2012.

[10] Rashidah Funke Olanrewaju, Muhammad Luqman Azzaki Mohd Radzi, Mariam Rehab, Fawwaz Eniola Fajingbesi, "Design and Fabrication of an Intelligent Walking Staff for Visually Impaired Subjects," IJEECS , vol. 10, pp.266270, 2018.

[11] Xu Bing, "Key Internet of Things Technology and Application Research,” TELKOMNIKA , vol. 12, pp.5599-5602, 2014.

[12] S.A. Ishak, H.Zainol Abidin, M.Muhamad, "Improving Medical Adherence using Smart Medicine Cabinet Monitoring System,"IJEECS , vol. 9, pp.164-169, 2018. 